\title{
A Three-Parameter Gompertz-Lindley Distribution: Its Properties
}

\section{and Applications}

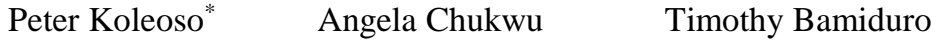 \\ Department of Statistics, University of Ibadan, Ibadan, Oyo State, Nigeria.
}

\begin{abstract}
This research proposed a three-parameter probability distribution called Gompertz-Lindley distribution using Gompertz generalized (Gompertz-G) family of distributions. The mathematical properties of the distribution such as moment, moment generating function, survival function and hazard function were derived. The parameters of the distribution were estimated using the method of maximum likelihood and the distribution was applied to model the strength of glass fibres. Gompertz-Lindley distribution performed best (AIC $=62.8537$ ) when compared with other generalizations of the Lindley distribution.
\end{abstract}

Keywords: Gompertz distribution, Lindley distribution, moment, survival function, hazard function

DOI: $10.7176 / \mathrm{MTM} / 9-4-04$

Publication date: April $30^{\text {th }} 2019$.

\section{Introduction}

There are several statistical distributions that have been useful in various fields such as medicine, economics, insurance, agriculture, finance, engineering, etc. The quest for more flexible models to model complex data sets has led to several new distributions that are obtained by generalizing the baseline distributions (Oguntunde et al. 2015). Lindley distribution has been known for its good performance generally in many applications most especially in modelling lifetime data sets (Shanker et al. 2017). The Lindley distribution introduced by Lindley (1958) in the context of Bayesian analysis as a counter example of fiducial statistics. The cumulative distribution function (C. D. F) and probability density function (P. D. F) are given below:

$$
G(x)=1-\left[1+\frac{\theta x}{\theta+1}\right] e^{-\theta x}
$$

and

$$
g(x)=\frac{\theta^{2}}{\theta+1}(1+x) e^{-\theta x}
$$

respectively, where $x>0, \theta>0$, and $\theta$ is the scale parameter of the distribution.

Several research works have been carried out in the past on Lindley distribution and its improvement for greater flexibility. Some of these can be found in the works of Shanker et al. (2017) that proposed a three-parameter Lindley distribution for modelling lifetime data, Shanker and Mishra (2014) which proposed two parameter Poisson-Lindley distribution, its properties and application to real life data set. Shanker and Mishra (2013b) proposed a two-parameter Lindley distribution. Zakerzadeh and Dolati (2009) introduced a three-parameter generalized Lindley distribution, its estimation and application to real life data. This research focuses on the improving the flexibility of Lindley distribution. 
This article is arranged in sections. Section 2 includes the methods which involve the distributional properties of the proposed Gompertz-Lindley distribution. Section 3 includes results and discussion, which involve application of the distribution to real life data. Section 4 is the conclusion.

\section{Methods}

This section describes the properties of the proposed Gompertz-Lindley distribution. For any continuous distribution with cumulative distribution function $G(x)$ and probability density function, $g(x)$, Alizadeh $e t$ al. (2017) proposed the Gompertz generalized (denoted as "Gompertz-G) family of distributions that provides greater flexibility in modelling of real data sets. The $c d f$ and $p d f$ of the Gompertz-G family are defined for any continuous distribution as:

$$
F(x)=\int_{0}^{-\log [1-G(x)]} \alpha e^{\beta t} e^{-\frac{\alpha}{\beta}\left(e^{\beta t}-1\right)} d t
$$

Solving the integral above, equation (3) yields the cdf and pdf of the Gompertz-G family as

$$
F(x)=1-e^{\frac{\alpha}{\beta}\left\{1-[1-G(x)]^{-\beta}\right\}}
$$

and

$$
f(x)=\alpha g(x)[1-G(x)]^{-\beta-1} e^{\frac{\alpha}{\beta}\left\{1-[1-G(x)]^{-\beta}\right\}}
$$

respectively, where $\mathrm{g}(x)$ and $\mathrm{G}(x)$ are the $p d f$ and $c d f$ of any continuous distribution to be generalized respectively.

The parameters, $\alpha>0$ and $\beta>0$ are the two additional shape parameters useful for making the kurtosis more flexible compared to the baseline model. These produce skewness for symmetrical distributions, generate distributions with symmetric, left-skewed, right-skewed, and reversed-J shaped and special models with all types of the hazard functions. This family of distribution will provide consistently better fits than other generated models under the same baseline distribution (Alizadeh et al., 2017).

Inserting equations (1) and (2) into equations (4) and (5) and simplifying, the cdf and pdf of the Gompertz-Lindley distribution are obtained as follows:

$$
\begin{gathered}
F(x)=1-e^{\frac{\alpha}{\beta}\left\{1-\left[1-\left(1-\left[1+\frac{\theta x}{\theta+1}\right] e^{-\theta x}\right)\right]^{-\beta}\right\}} \\
F(x)=1-e^{\frac{\alpha}{\beta}\left\{1-\left[\left[1+\frac{\theta x}{\theta+1}\right] e^{-\theta x}\right]^{-\beta}\right\}}
\end{gathered}
$$


And

$$
\begin{aligned}
& \left.f(x)=\alpha \frac{\theta^{2}}{\theta+1}(1+x) e^{-\theta x}\left[1-\left(1-\left[1+\frac{\theta x}{\theta+1}\right] e^{-\theta x}\right)\right]^{-\beta-1} \frac{\alpha}{\beta} e^{\left\{-\left[1-\left[1-\left[1+\frac{\theta x}{\theta+1}\right] e^{-\theta x}\right)\right]^{-\beta}\right.}\right\} \\
& f(x)=\alpha \frac{\theta^{2}}{\theta+1}(1+x) e^{-\theta x\left[\left[1+\frac{\theta x}{\theta+1}\right]\right.} e^{-\theta x]^{-\beta-1}} e^{\left.\frac{\alpha}{\beta}\left\{1-\left[1+\frac{\theta x}{\theta+1}\right] e^{-\theta x}\right]^{-\beta}\right\}}
\end{aligned}
$$

respectively. Hence equations (6) and (7) are the cdf and pdf of the Gompertz-Lindley distribution (GLD) where $\theta>0$ is a scale parameter while $\alpha>0$ and $\beta>0$ are the extra shape parameters.

\subsection{Properties of the Gompertz-Lindley Distribution}

\subsubsection{Valid Probability Density Function}

The above distribution is valid if and only if $\int_{-\infty}^{\infty} f(x) d x=1$

\section{Proof:}

$$
\begin{aligned}
f(x) & \left.=\alpha \frac{\theta^{2}}{\theta+1}(1+x) e^{-\theta x}\left[\left[1+\frac{\theta x}{\theta+1}\right] e^{-\theta x}\right]^{-\beta-1} e^{\frac{\alpha}{\beta}\left\{1-\left[\left[1+\frac{\theta x}{\theta+1}\right] e^{-\theta x}\right]^{-\beta}\right.}\right\} \\
\int_{-\infty}^{\infty} f(x) d x & =\int_{0}^{\infty} \alpha \frac{\theta^{2}}{\theta+1}(1+x) e^{-\theta x}\left[\left[1+\frac{\theta x}{\theta+1}\right] e^{-\theta x}\right]^{-\beta-1} e^{\left.\frac{\alpha}{\beta}\left\{1-\left[1+\frac{\theta x}{\theta+1}\right] e^{-\theta x}\right]^{-\beta}\right\}} d x
\end{aligned}
$$

let

$\left.y=e^{\frac{\alpha}{\beta}\left\{1-\left[\left[1+\frac{\theta x}{\theta+1}\right] e^{-\theta x}\right]^{-\beta}\right\}}=e^{u}, u=\frac{\alpha}{\beta}\left\{1-\left[1+\frac{\theta x}{\theta+1}\right] e^{-\theta x}\right]^{-\beta}\right\}=\frac{\alpha}{\beta}-\frac{\alpha}{\beta} t^{-\beta}$ and $t=\left[1+\frac{\theta x}{\theta+1}\right] e^{-\theta x}$

then 
Mathematical Theory and Modeling

www.iiste.org

ISSN 2224-5804 (Paper) ISSN 2225-0522 (Online) DOI: 10.7176/MTM

Vol.9, No.4, 2019

pISTE

$$
\begin{gathered}
\int_{-\infty}^{\infty} f(x) d x=\int_{0}^{\infty} \frac{\alpha \theta^{2}}{\theta+1}(1+x) e^{-\theta x} t^{-\beta-1} e^{u} d x \\
\frac{d y}{d x}=\frac{d y}{d u} \times \frac{d u}{d t} \times \frac{d t}{d x} \\
\frac{d y}{d u}=e^{u}, \frac{d u}{d t}=\alpha t^{-\beta-1}, \text { and } \frac{d t}{d x}=-\frac{\theta^{2}}{\theta+1}(1+x) e^{-\theta x}
\end{gathered}
$$

Hence

$$
\begin{gathered}
\frac{d y}{d x}=e^{u} \times \alpha t^{-\beta-1} \times-\frac{\theta^{2}}{\theta+1}(1+x) e^{-\theta x} \\
\frac{d y}{d x}=-\frac{\alpha \theta^{2}}{\theta+1}(1+x) e^{-\theta x} t^{-\beta-1} e^{u}
\end{gathered}
$$

and

$$
d x=-\frac{(\theta+1) d y}{\alpha \theta^{2}(1+x) e^{-\theta x} t^{-\beta-1} e^{u}}
$$

Therefore, substituting for $d x$ in equation (8) and simplifying, we obtain

$$
\int_{-\infty}^{\infty} f(x) d x=-\int_{0}^{\infty} d y=-[y]_{-\infty}^{\infty}
$$

32 


$$
\begin{aligned}
& \int_{-\infty}^{\infty} f(x) d x=-\left[e^{\frac{\alpha}{\beta}\left\{1-\left[\left[1+\frac{\theta x}{\theta+1}\right] e^{-\theta x}\right]^{-\beta}\right\}}\right]_{0}^{\infty} \\
& \int_{-\infty}^{\infty} f(x) d x=-\left[e^{\frac{\alpha}{\beta}\left\{1-\left[\left[1+\frac{\theta(\infty)}{\theta+1}\right] e^{-\theta(\infty)}\right]^{-\beta}\right\}}-e^{\frac{\alpha}{\beta}\left\{1-\left[\left[1+\frac{\theta(0)}{\theta+1}\right] e^{-\theta(0)}\right]^{-\beta}\right\}}\right]=-[0-1]=0+1=1
\end{aligned}
$$

Hence, equation (9) is a proof that the model in equation (7) is a valid probability density function.

The following is a graphical representation of the $p d f$ and $c d f$ of the Gompertz-Lindley distribution. Given some values for the parameters $\theta, \alpha$ and $\beta$, possible plots for the $p d f$ and the $c d f$ of the Gompertz-Lindley distribution are shown in the figures below:

\section{Pdf of GLD}

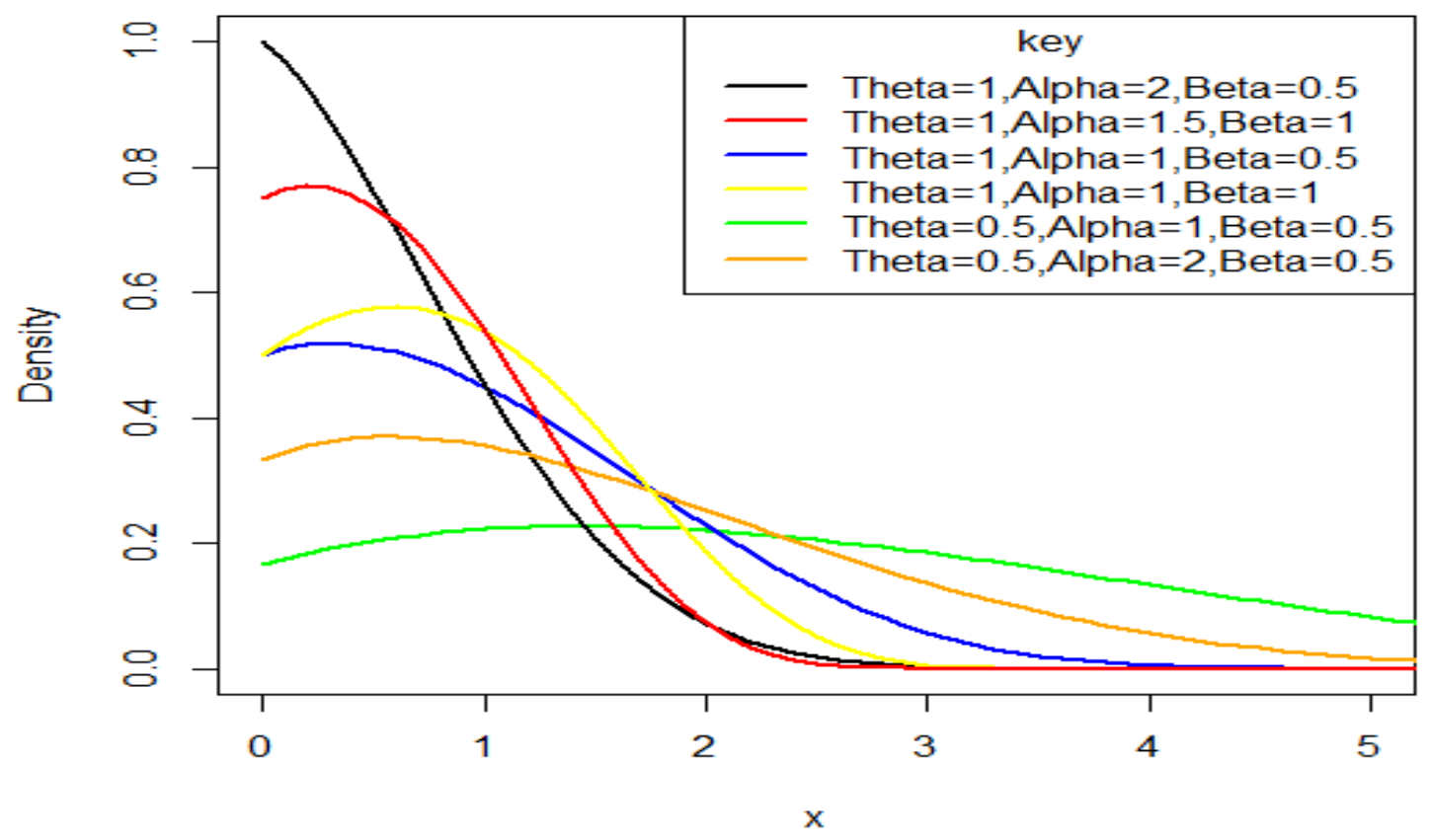

Figure 1. Probability Density Function plots of Gompertz-Lindley Distribution 


\section{Cdf of GLD}

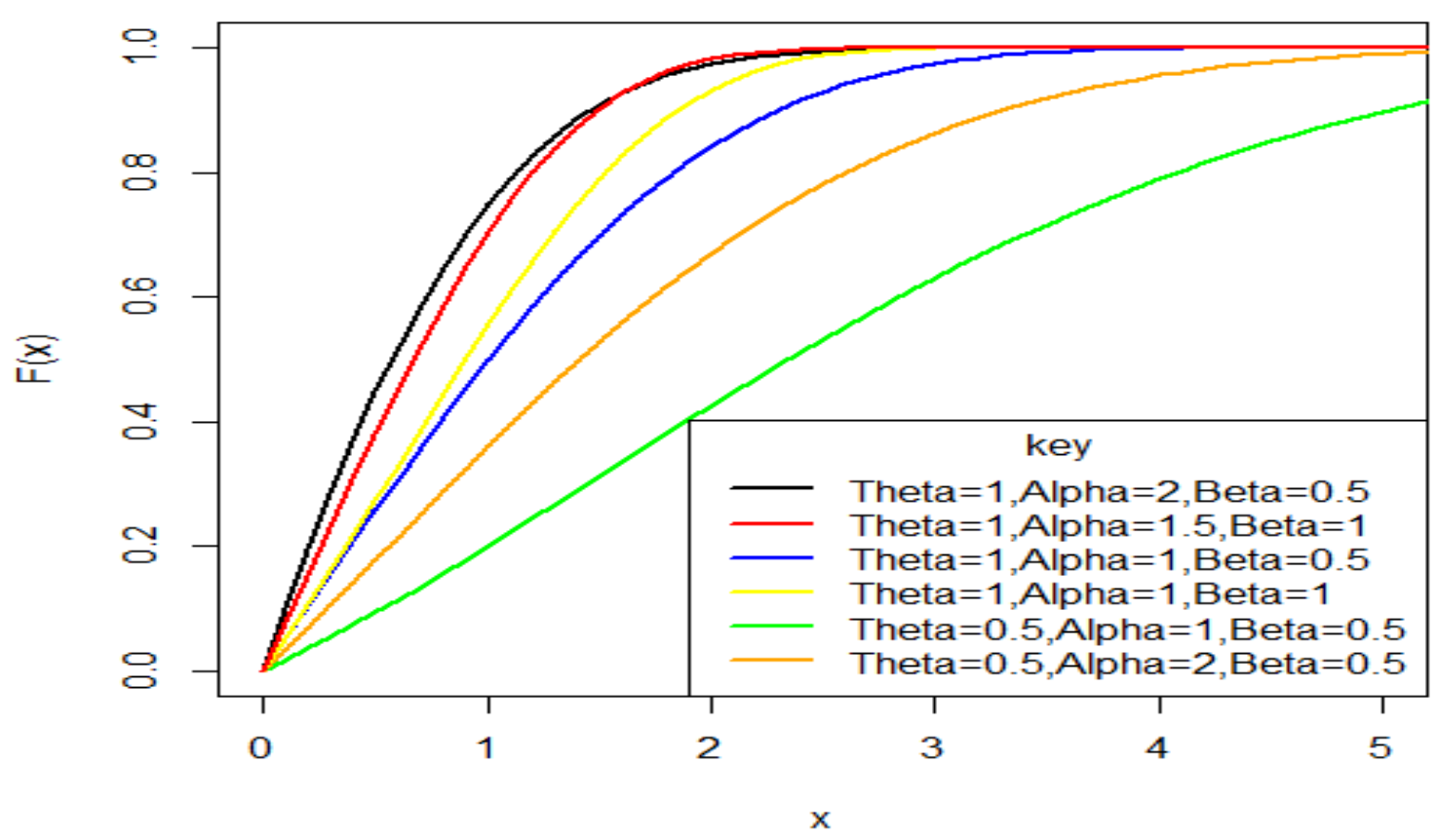

Figure.2. Cumulative Distribution Function plots of Gompertz-Lindley Distribution

\subsubsection{Moment}

Let $\mathrm{X}$ denote a continuous random variable, the $n^{\text {th }}$ moment of $X$ is given by;

$$
\boldsymbol{\mu}_{n}^{\prime}=E\left(X^{n}\right)=\int_{0}^{\infty} x^{n} f(x) d x
$$

Take $f(x)$ to be the $p d f$ of the Gompertz-Lindley distribution as given in equation (7) and substituting in equation

(10), to get the moment of the distribution.

$$
f(x)=\alpha \frac{\theta^{2}}{\theta+1}(1+x) e^{-\theta x\left[\left[1+\frac{\theta x}{\theta+1}\right] e^{-\theta x}\right]^{-\beta-1} \frac{\alpha}{\beta}\left\{1-\left[\left[1+\frac{\theta x}{\theta+1}\right] e^{-\theta x}\right]^{-\beta}\right\}}
$$

Consider the following expansion and simplification of the pdf

By expanding the exponential term in (11) using power series, we obtain: 


$$
e^{\left.\frac{\alpha}{\beta}\left\{1-\left[1+\frac{\theta x}{\theta+1}\right] e^{-\theta x}\right]^{-\beta}\right\}}=\sum_{i=0}^{\infty} \frac{\alpha^{i}}{i ! \beta^{i}}\left[1-\left[\left[1+\frac{\theta x}{\theta+1}\right] e^{-\theta x}\right]^{-\beta}\right]^{i}
$$

Making use of the result in (12) above, equation (11) becomes

$$
f(x)=\frac{\alpha \theta^{2}}{\theta+1} \sum_{i=0}^{\infty} \frac{\alpha^{i}}{i ! \beta^{i}}(1+x) e^{-\theta x}\left[\left[1+\frac{\theta x}{\theta+1}\right] e^{-\theta x}\right]^{-\beta-1}\left[1-\left[\left[1+\frac{\theta x}{\theta+1}\right] e^{-\theta x}\right]^{-\beta}\right]^{i}
$$

Also, using the generalized binomial theorem, we can write the last term from the above result as:

$$
\left(1-\left[\left[1+\frac{\theta x}{\theta+1}\right] e^{-\theta x}\right]^{-\beta}\right)^{i}=\sum_{j=0}^{\infty}(-1)^{j}\left(\begin{array}{l}
i \\
j
\end{array}\right)\left(\left[1+\frac{\theta x}{\theta+1}\right] e^{-\theta x}\right)^{-\beta j}
$$

Making use of the result in (14) above, equation (13) becomes

$$
\begin{aligned}
& f(x)=\frac{\alpha \theta^{2}}{\theta+1} \sum_{i=0}^{\infty} \sum_{j=0}^{\infty} \frac{\alpha^{i}(-1)^{j}}{i ! \beta^{i}}\left(\begin{array}{l}
i \\
j
\end{array}\right)(1+x) e^{-\theta x}\left[\left(1+\frac{\theta x}{\theta+1}\right) e^{-\theta x}\right]^{-[\beta(j+1)+1]} \\
& f(x)=\frac{\alpha \theta^{2}}{\theta+1} \sum_{i=0}^{\infty} \sum_{j=0}^{\infty} \frac{\alpha^{i}(-1)^{j}}{i ! \beta^{i}}\left(\begin{array}{l}
i \\
j
\end{array}\right)(1+x) e^{\theta \beta(j+1) x}\left[1+\frac{\theta x}{\theta+1}\right]^{-[\beta(j+1)+1]} \\
& f(x)=\frac{\alpha \theta^{2}}{\theta+1} \sum_{i=0}^{\infty} \sum_{j=0}^{\infty} \frac{\alpha^{i}(-1)^{j}}{i ! \beta^{i}}\left(\begin{array}{l}
i \\
j
\end{array}\right)(1+x) e^{\theta \beta(j+1) x}\left[\left(1+\frac{\theta x}{\theta+1}\right)^{\beta(j+1)+1}\right]^{-1}
\end{aligned}
$$

Using power series expansion on the last term in equation (15), it gives:

$$
\begin{aligned}
& \left(1+\frac{\theta x}{\theta+1}\right)^{\beta(j+1)+1}=\sum_{k=0}^{\infty}\left(\begin{array}{c}
\beta(j+1)+1 \\
k
\end{array}\right)\left(\frac{\theta x}{\theta+1}\right)^{k} \\
& \left(1+\frac{\theta x}{\theta+1}\right)^{\beta(j+1)+1}=\sum_{k=0}^{\infty}\left(\begin{array}{c}
\beta(j+1)+1 \\
k
\end{array}\right)\left(\frac{\theta}{\theta+1}\right)^{k} x^{k}
\end{aligned}
$$

Now, substituting equation (16), the power series expansion in equation (15) above gives: 
$f(x)=\frac{\alpha \theta^{2}}{\theta+1} \sum_{i=0}^{\infty} \sum_{j=0}^{\infty} \frac{\alpha^{i}(-1)^{j}}{i ! \beta^{i}}\left(\begin{array}{c}i \\ j\end{array}\right)\left[\sum_{k=0}^{\infty}\left(\begin{array}{c}\beta(j+1)+1 \\ k\end{array}\right)\left(\frac{\theta}{\theta+1}\right)^{k}\right]^{-1} x^{-k}(1+x) e^{\theta \beta(j+1) x}$

Simplifying equation results in the following:

$$
\begin{gathered}
f(x)=\frac{\alpha \theta^{2}}{\theta+1} \sum_{i=0}^{\infty} \sum_{j=0}^{\infty} \frac{\alpha^{i}(-1)^{j}}{i ! \beta^{i}}(i)\left[\sum_{j=0}^{\infty}\left(\begin{array}{c}
\beta(j+1)+1 \\
k
\end{array}\right)\left(\frac{\theta}{\theta+1}\right)^{k}\right]^{-1} x^{-k}(1+x) e^{\theta \beta(j+1) x} \\
f(x)=\eta_{i, j, k} x^{-k}(1+x) e^{\theta \beta(j+1) x}
\end{gathered}
$$

Where

$$
\eta_{i, j, k}=\frac{\alpha \theta^{2}}{\theta+1} \sum_{i=0}^{\infty} \sum_{j=0}^{\infty} \frac{\alpha^{i}(-1)^{j}}{i ! \beta^{i}}\left(\begin{array}{c}
i \\
j
\end{array}\right)\left[\sum_{k=0}^{\infty}\left(\begin{array}{c}
\beta(j+1)+1 \\
k
\end{array}\right)\left(\frac{\theta}{\theta+1}\right)^{k}\right]^{-1}
$$

Hence,

$$
\begin{aligned}
& \mu_{n}^{\prime}=E\left(X^{n}\right)=\int_{0}^{\infty} x^{n} f(x) d x=\int_{0}^{\infty} \eta_{i, j, k} x^{n-k}(1+x) e^{\theta \beta(j+1) x} d x \\
& \mu_{n}^{\prime}=E\left[X^{n}\right]=\int_{0}^{\infty} x^{n} f(x) d x=\eta_{i, j, k}\left[\int_{0}^{\infty} x^{n-k} e^{\theta \beta(j+1) x} d x+\int_{0}^{\infty} x^{n-k+1} e^{\theta \beta(j+1) x} d x\right]
\end{aligned}
$$

Solving equation (19); it gives the nth moment of $\mathrm{X}$ for the Gompertz-Lindley distribution as follows:

$$
E\left[X^{n}\right]=\mu_{n}^{\prime}=\eta_{i, j, k}\left[\left(-\frac{1}{\theta \beta(j+1)}\right)^{n-k+1} \Gamma(n-k+1)+\left(-\frac{1}{\theta \beta(j+1)}\right)^{n-k+2} \Gamma(n-k+2)\right]_{(20)}
$$

\subsubsection{Moment Generating Function}

The $m g f$ of a random variable $X$ can be obtained by 


$$
M_{x}(t)=E\left[e^{t x}\right]=\int_{0}^{\infty} e^{t x} f(x) d x
$$

Simplifying the integral in (21), it gives;

$$
\begin{gathered}
M_{x}(t)=E\left[e^{t x}\right]=E\left(\sum_{n=0}^{\infty} \frac{(t x)^{n}}{n !}\right)=\sum_{n=0}^{\infty} \frac{t^{n}}{n !} \mu_{n} \\
M_{x}(t)=\sum_{n=0}^{\infty} \frac{t^{n}}{n !}\left[\eta_{i, j, k}\left[\left(-\frac{1}{\theta \beta(j+1)}\right)^{n-k+1} \Gamma(n-k+1)+\left(-\frac{1}{\theta \beta(j+1)}\right)^{n-k+2} \Gamma(n-k+2)\right]\right]
\end{gathered}
$$

\subsubsection{Survival Function}

Survival function is the likelihood that a system or an individual will not fail after a given time. Mathematically, it is given by:

$$
S(x)=1-F(x)
$$

Where $F(x)$ is $c d f$ of the Gompertz-Lindley distribution.

$$
S(x)=e^{\frac{\alpha}{\beta}\left\{1-\left[\left[1+\frac{\theta x}{\theta+1}\right] e^{-\theta x}\right]^{-\beta}\right\}}
$$

Below is a plot of the survival functions at chosen parameter values in figure 3: 


\section{Survival function of GLD}

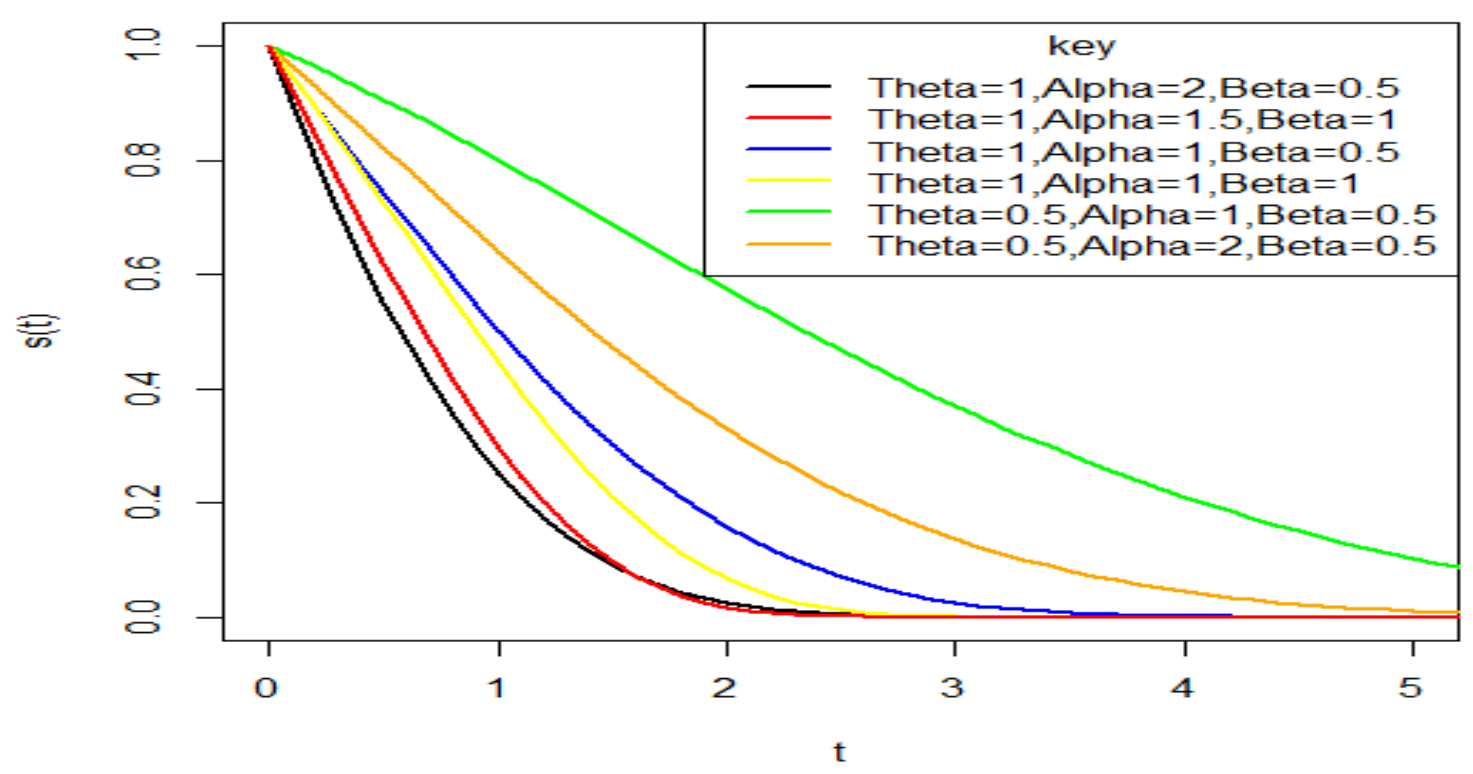

Figure 3. Survival function plots of Gompertz-Lindley Distribution

\subsubsection{Hazard Function}

The hazard function which is the failure rate is defined mathematically as;

$$
h(x)=\frac{f(x)}{1-F(x)}=\frac{f(x)}{S(x)}
$$

Taking $f(x)$ and $F(x)$ to be the $p d f$ and $c d f$ of the proposed Gompertz-Lindley distribution, the hazard function is given as:

$$
\begin{gathered}
h(x)=\frac{\frac{\alpha \theta^{2}}{\theta+1}(1+x) e^{-\theta x}\left[\left[1+\frac{\theta x}{\theta+1}\right] e^{-\theta x}\right]^{-\beta-1} e^{\frac{\alpha}{\beta}}\left\{1-\left[\left[1+\frac{\theta x}{\theta+1}\right] e^{-\theta x}\right]^{-\beta}\right\}}{\left(1-\left[1-e^{\frac{\alpha}{\beta}\left\{1\left[\left[1+\frac{\theta x}{\theta+1}\right] e^{-\theta x}\right]^{-\beta}\right\}}\right]\right)} \\
h(x)=\frac{\alpha \theta^{2}}{\theta+1}(1+x) e^{-\theta x}\left[\left[1+\frac{\theta x}{\theta+1}\right] e^{-\theta x}\right]^{-\beta-1}
\end{gathered}
$$

The following is a plot of the hazard functions at chosen parameter values in figure 4 . 


\section{Failure rate of GLD}

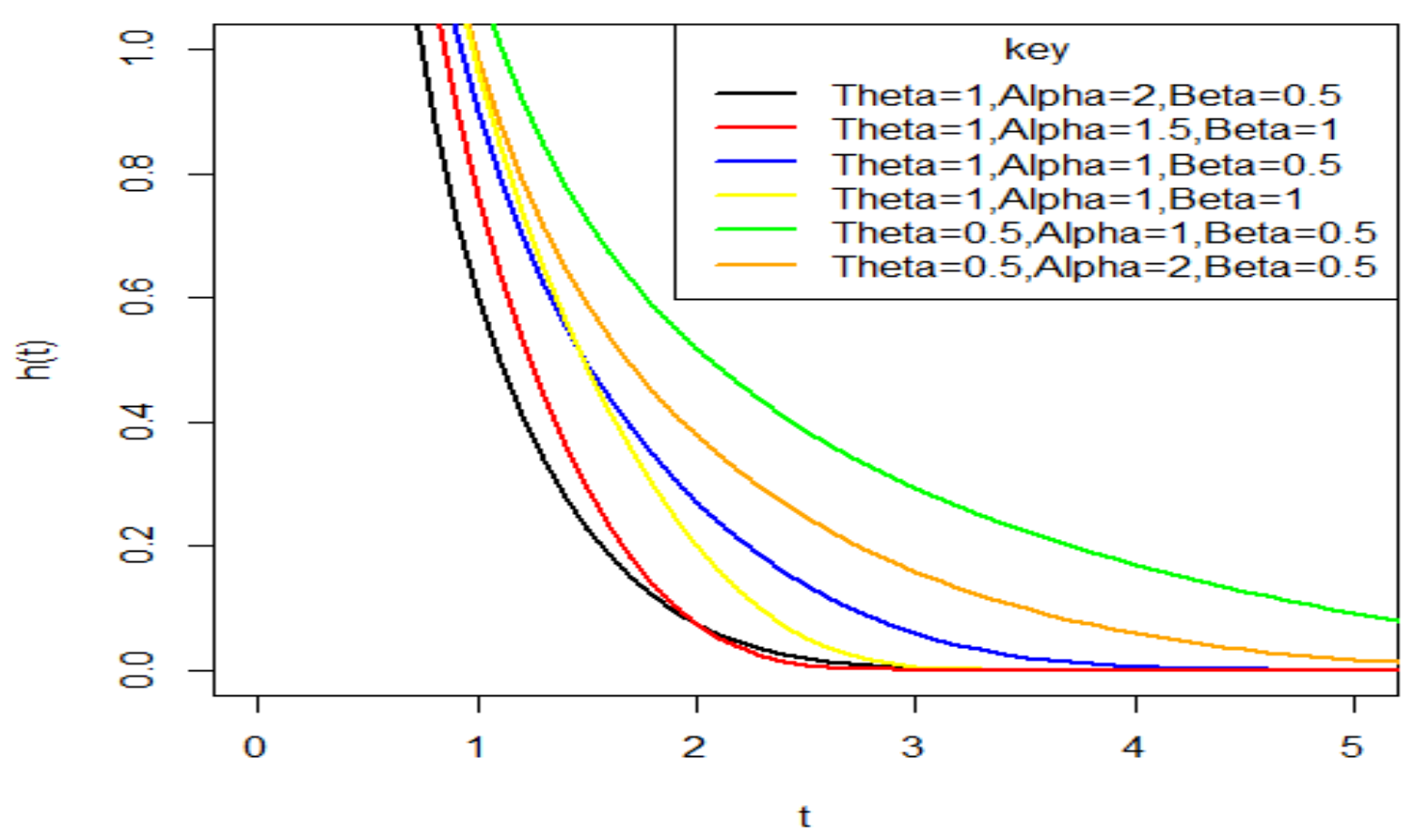

Figure 4. The hazard function plots of Gompertz-Lindley distribution

\subsubsection{Estimation of Parameters}

Let $X_{1}, X_{2}, \ldots ., X_{n}$ be a sample of size 'n' independently and identically distributed random variables from the Gompertz-Lindley Distribution (GLD) with unknown parameters $\alpha, \beta$ and $\theta$ defined previously. The $p d f$ of the $G L D$ is given from (7) as;

$$
f(x)=\frac{\alpha \theta^{2}}{\theta+1}(1+x) e^{-\theta x}\left[\left[1+\frac{\theta x}{\theta+1}\right] e^{-\theta x}\right]^{-\beta-1} e^{\left.\frac{\alpha}{\beta}\left\{1-\left[1+\frac{\theta x}{\theta+1}\right] e^{-\theta x}\right]^{-\beta}\right\}}
$$

Using the Maximum Likelihood Estimation method, the likelihood function is given by;

$$
L(\underline{X} \mid \alpha, \beta, \theta)=\frac{\left(\alpha \theta^{2}\right)^{n} \prod_{i=1}^{n}\left\{\left(1+x_{i}\right) e^{-\theta x_{i}}\right\} \prod_{i=1}^{n}\left\{\left[1+\frac{\theta x_{i}}{\theta+1}\right] e^{-\theta x_{i}}\right\}^{-\beta-1} \frac{\alpha}{\beta} e^{\frac{\alpha}{\beta}\left\{1-1\left[\left[1+\frac{\theta x_{i}}{\theta+1}\right] e^{-\theta x_{i}}\right]^{-\beta}\right\}}}{(\theta+1)^{n}}
$$

and the $\log$-likelihood function, $l=\log L(\underline{X} \mid \alpha, \beta, \theta)$ is given by; 


$$
l=n \log \alpha+2 n \log \theta-n \log (\theta+1)+\sum_{i=1}^{n} \log \left(1+x_{i}\right)-\theta \sum_{i=1}^{n} x_{i}-(\beta+1) \sum_{i=1}^{n} \log \left\{\left[1+\frac{\theta x_{i}}{\theta+1}\right] e^{-\theta x_{i}}\right\}+\frac{\alpha}{\beta} \sum_{i=1}^{n}\left\{1-\left[\left[1+\frac{\theta x_{i}}{\theta+1}\right] e^{-\theta x_{i}}\right]^{-\beta}\right\}
$$

Differentiating $\boldsymbol{l}$ partially with respect to $\alpha, \beta$ and $\theta$ respectively gives the maximum likelihood estimates of the model parameters. These estimates can be obtained using the R programming software in the presence of data sets.

\section{Results and Discussion}

In this section, the proposed Gompertz-Lindley distribution was applied to a data set and compared with some extensions of the Lindley distribution. The performance was compared with some other generalizations of the Lindley distribution such as Generalized Lindley distribution $(G e n L n D)$, a three-parameter Lindley distribution (ATPLnD), Transmuted two-parameter Lindley distribution (TTPLnD), Transmuted Lindley distribution (TLnD) and the Lindley distribution $(L n D)$. The data set used is on the strengths of $1.5 \mathrm{~cm}$ glass fibres initially collected by members of staff at the UK National Physical Laboratory. It has been used by Mansour et al. (2018), Barreto-Souza et al. (2011), Bourguignon et al. (2014), Oguntunde et al. (2015) as well as Smith and Naylor (1987). The descriptive statistics and the performance of the models are given in the tables below:

Table 1. Descriptive Statistics for the Glass Fibres data set

\begin{tabular}{|l|l|l|l|l|l|l|l|l|l|}
\hline n & Minimum & $Q_{1}$ & Median & $Q_{3}$ & Mean & Maximum & Variance & Skewness & Kurtosis \\
\hline 63 & 0.550 & 1.375 & 1.590 & 1.685 & 1.507 & 2.240 & 0.105 & -0.8786 & 3.9238 \\
\hline
\end{tabular}


Table 2. Model Performances

\begin{tabular}{|l|l|l|l|l|l|l|l|}
\hline Distribution & $\begin{array}{l}\text { Parameter } \\
\text { estimates }\end{array}$ & $\begin{array}{l}\text {-ll=(-log } \\
\text {-likeliho } \\
\text { od } \\
\text { value })\end{array}$ & AIC & CAIC & BIC & HQIC & $\begin{array}{l}\text { Rank of } \\
\text { model } \\
\text { perform } \\
\text { ance }\end{array}$ \\
\hline 1.GLD & $\begin{array}{l}\widetilde{\boldsymbol{\theta}}=0.5724 \\
\widehat{\boldsymbol{\alpha}}=0.3188 \\
\widetilde{\boldsymbol{\beta}}=5.9338\end{array}$ & 28.4268 & 62.8537 & 63.2605 & 69.2831 & 65.3824 & 1 \\
\hline 2.GenLnD & $\begin{array}{l}\widetilde{\boldsymbol{\theta}}=2.2962 \\
\widehat{\boldsymbol{\alpha}}=8.8328\end{array}$ & 36.8119 & 77.6237 & 77.8237 & 81.9100 & 79.3095 & 2 \\
\hline 3.TTPLnD & $\begin{array}{l}\widetilde{\boldsymbol{\theta}}=1.7837 \\
\widehat{\boldsymbol{\alpha}}=9.5847 \\
\widetilde{\boldsymbol{\beta}}=-0.9779\end{array}$ & 53.8047 & 113.6095 & 114.0162 & 120.0389 & 116.1382 & 3 \\
\hline 4. ATPLnD & $\begin{array}{l}\widetilde{\boldsymbol{\theta}}=1.2473 \\
\widehat{\boldsymbol{\alpha}}=0.6207 \\
\widetilde{\boldsymbol{\beta}}=7.0493\end{array}$ & 69.3115 & 144.6229 & 145.0297 & 151.0523 & 147.1516 & 5 \\
\hline $\begin{array}{l}\widetilde{\boldsymbol{\theta}}=1.3216 \\
\widehat{\boldsymbol{\lambda}}=-0.9998\end{array}$ & 62.6736 & 129.3471 & 129.5471 & 133.6334 & 131.0329 & 4 \\
\hline $\begin{array}{l}\widetilde{\boldsymbol{\theta}}=0.9961 \\
\text { 5. TLnD }\end{array}$ & 81.2784 & 164.5569 & 164.6225 & 166.7000 & 165.3998 & 6 \\
\hline 6. LnD & & & & & & \\
\hline Bayesian Information Criterion and HQIC $=$ Hannan Quinn Information Criterion & & \\
\hline
\end{tabular}

The data size for the data set on the strengths of glass fibres from Table 1 was 63 with the mean and median being approximately the same $($ mean $=1.507$, median $=1.590)$. The proposed Gompertz-Lindley distribution performed best in modelling the strength of glass fibres (AIC $=62.8537$ ), followed by Generalized Lindley distribution (AIC $=77.6237$ ). The Lindley distribution had the least performance with the highest information criterion values. The performance of the model was based on the information criterion values, that is, the lower the information criterion value, the better the model.

The research outcome supports the notion that generalizing a baseline distribution produces a more flexible distribution than the baseline distribution itself (Oguntunde et. al. 2015).

\section{Conclusion}

The three-parameter Gompertz-Lindley distribution was proposed in this article as an improvement on the Lindley distribution. The distribution provided greater flexibility in modelling real life data. The distribution also performed best when compared with other generalizations of the Lindley distribution to fit the strength of glass fibres.

\section{References}

Alizadeh, M., Cordeiro, G. M., Pinho, L. G. B. et al. (2017). "The Gompertz-G Family of Distributions". Journal of Statistical Theory and Practice 11(1):179-207. doi:10.1080/15598608.2016.1267668. 
Barreto-Souza, W., Cordeiro, G. M. and Simas, A. B. (2011). "Some Results for Beta Frechet Distribution". Communications in Statistics - Theory and Methods 40(5):798-811.

Bourguignon M., Silva, R. B. and Cordeiro, G. M. (2014). “The Weibull-G Family of Probability Distributions". Journal of Data Science 12:53-68.

Lindley, D. V. (1958). "Fiducial Distributions and Bayes' Theorem”. Journal of Royal Statistical Society, Series B (Methodological) 20(1):102-107.

Mansour, M., Aryal, G., Afify, A. Z. et al. (2018). “The Kumaraswamy Exponentiated Frechet Distribution. Pakistan Journal of Statistics 34(3):177-193.

Oguntunde, P. E., Balogun, O. S., Okagbue, H. I. et al. (2015). “The Weibull-Exponential Distribution: its Properties and Applications". Journal of Applied Sciences 15(11):1305-1311.

Shanker, R. and Mishra, A. (2013b). “A Two-parameter Lindley Distribution”. Statistics in Transition-New Series 14(1):45-56.

Shanker, R. and Mishra, A. (2014). “A Two-parameter Poisson-Lindley distribution”. International Journal of Statistics and Systems 9(1):79-85.

Shanker, R., Shukla, K. K., Shanker, R. et al. (2017). “A Three-parameter Lindley distribution”. American Journal of Mathematics and Statistics 7(1):15-26.

Smith, R. L. and Naylor, J. C. (1987). "A Comparison of Maximum Likelihood and Bayesian Estimators for the Three-Parameter Weibull Distribution”. Journal of Royal Statistical Society, Series C (Applied Statistics) 36(3):358-369.

Zakerzadeh, H. and Dolati, A. (2009). “Generalized Lindley Distribution”. Journal of Mathematical Extension $3(2): 13-25$. 